Erratum

\title{
An IL-7 splicing-defect lymphopenia mouse model revealed by genome-wide mutagenesis
}

\author{
Hong-Wen Huang ${ }^{1}$, Yun-Jung Chiang ${ }^{1}$, Shuen-Iu Hung ${ }^{2}$, Chung-Leung Li $^{3}$ \& Jeffrey \\ Jong-Young Yen ${ }^{1 * *}$ \\ ${ }^{1}$ Institute of Biomedical Sciences, Academia Sinica, No. 128, Sec.2, Yen-Jiou-Yuan Rd., Taipei, 11529, \\ Taiwan; ${ }^{2}$ Genotyping Center, Genomics Research Center, Academia Sinica, Taipei, 11529, Taiwan; ${ }^{3}$ Stem \\ Cell Program, Institute of Cellular \& Organismic Biology/Genomics Research Center, Academia Sinica, \\ Taipei, 11529, Taiwan
}

The authors requested the following corrections for the article entitled 'An IL-7 splicing-defect lymphopenia mouse model revealed by genomewide mutagenesis' (J Biomed Sci 2006; Dec 7; Epub ahead of print):

The authorship, "Hong-Wen Huang', YunJung Chiang ${ }^{1}$, Shuen-Iu Hung ${ }^{2}$, Chung-Leung Li $^{3}$ and Jeffrey Jong-Young Yen ${ }^{1, *}$ " should read "Hong-Wen Huang 1,4, Yun-Jung Chiang ${ }^{1,4}$, Shuen-Iu Hung ${ }^{2}$, Chung-Leung $\mathrm{Li}^{3}$ and Jeffrey JongYoung Yen ${ }^{1, *}$, , and the footnote of the authorship, "Institute of Biomedical Sciences, Academia Sinica, No. 128, Sec.2, Yen-Jiou-Yuan Rd., Taipei, 11529, Taiwan; ${ }^{2}$ Genotyping Center,
Genomics Research Center, Academia Sinica, Taipei, 11529, Taiwan; ${ }^{3}$ Stem Cell Program, Institute of Cellular \& Organismic Biology/Genomics Research Center, Academia Sinica, Taipei, 11529, Taiwan" should read "Institute of Biomedical Sciences, Academia Sinica, No. 128, Sec.2, YenJiou-Yuan Rd., Taipei, 11529 , Taiwan; ${ }^{2}$ Genotyping Center, Genomics Research Center, Academia Sinica, Taipei, 11529, Taiwan; ${ }^{3}$ Stem Cell Program, Institute of Cellular \& Organismic Biology/ Genomics Research Center, Academia Sinica, Taipei, 11529, Taiwan; ${ }^{4}$ The first two authors contribute to this article equally".

The first two authors contribute to this article equally.

*To whom correspondence should be addressed.

The online version of the original article can be found under doi:10.1007/s11373-006-9135-1 